\title{
Verfassungstheorien politischer
}

\section{Antipoden: Otto Kirchheimer und Carl Schmitt}

Kirchheimer hacte 1928 bei Car! Schmict in Bonn promoviert mit dem Thema "Zur Staststheoric des Sozialismus und Boischewismus '. Die Dissertation greift jene Themenseränge auf, die Schmirc in der Scudie "Die Dikrasur $\alpha^{2}$, in den zu dem Band -Polirische Theologies'zusammengestellıen Auísätzen zur Souveränicätstheorie und in der Parlamentarismusschrift erarbeirer haste. Nun ist es gewiß nicht ungewöhnlich, daß ein Promovend aus dem wissenschaftlichen Werk seines Dokrorvacers zitier, immerhin ist die ins Auge springende Affinitär in der Analyse und Einorúnung sozialer Bewegungen und politischer Prinzipien bemerkenswert. So geht die Disseration, ganz in Ubereinstimmung mit dem materialen Demokraciebegriff Schmists, von der Fragwürdigkeit einer Verbürgerung gesellschafriicher Homogenicät über das Medium des Nacionalbewußeseins aus, nachdem der kapiralistische Produktionsprozeß die Narion in zwei feindliche Klassen aufgespalten hat. Die Oprion für das Zusammenleben der sozialen Klassen efordere nun die Konzeprion einer formalen Demokrarie auf der Grundlage eines Klassengleichgewichts.' Dabei teilt Kirchheimer die beispielhaft in der Souveränitätstheorie der - Polizischen Theologie entwickelte Skepsis hinsichtlich der Daverhaftigkeir eines jeden sozialen Gleichgewichtsmodells. Die Politik der Balance ist nach Schmitr charakteristisch für den bürgerlichen Liberalismus, der um ein labiles Gleichgewichr zwischen der Arbeiterklasse und den traditionellen Machrträgern Monarchie, Adel, Armee, Kirche zum Zwecke der Sicherung bürgerlicher Herrschaft bemühs ist. ${ }^{6}$ Kirchheimer überträgt dieses Modell mirsamt seiner Konnotationen aut die sozialdemokrarische Arbeiterbewegung, die in der "Theorie vom doppeiten Fortschritr" die politische Legitimation für ihren Emanziparionskampf unter den Bedingungen der formalen Demokratie entworfen habe. Weil das sterige Wachstum der kapiralistischen Wirtschaft norwendig vom Fortschritr in der Entricklung der Menschheir zu mehr Humanitär beğleitet werde, vermag der sozialdemokracische Poliviker zu 'glauben an die Möglichkeit friedlicher Mehrheitsbildung in der formalen Demokratie und zu verrauen auf den .Kampl organisierrer, aufgeklärter Massen voll Stecigkeir und Besonnenheit. u'

I Auszugsweise wirderabgedrucks in: Kirchheimer, Von der Weimarer Republik zum Faschissnus: Die Auflösung der demakravischen Rechisordnung, Frankfun am Main 1976. S. 32 ff.

2 Schrik, Die Dikratur. ). Aull, Berlin 196, (Ersuull. 1921).

3 Ders., Polixische Theologie, München 191+ (Ersizufl. 1921).

4 Ders.. Die geistesgeschicheliche Lage des heutigen Parlancnesismus, +. Auh.. Berlin 1969 (Ersuufl. 1913).

s Kinchheimer, FN I, S. 35. Schmist hatte die an die Gleichgrwiehrsthese ansezzenden Uberlegungen Kinchheimers als -sehr beachienswere (!) sizis- und verlassungstheorctische Konstruktion. gelobr. Schmirt, Des Hüree der Veriassung, 2. Auf, Beplin 1969, S. 141 (Erseaufl. 19ur).

6 Ygl. Schmius Ausiührungen im f. Kapirel der Poluisehen Theologic, S. 67 If., .Zur Seaztsphilosophie der Gegencevolucion.

, Kirchineimer. FN I. S. 19 \%. 
Schmiu hat gegen dic liberalc Politik der Balance und ihren Glauben an den Ausgleich gesellschaldicher Widersprüche im servigen Gesprächa des Parlamentarismus Jen spanischen Gegenrevolutionär Donoso Conćs als Zeugen aulgerufen.' Donoso habe die liberalc Taktik des lachenden Dricten ais schlauen Schrindel der Bourgeoisie durchschaur und als illusionäres Ausweichmanöver vor der historisch unumgehbaren Entscheidung zwischen proiccarischer ×Dikiacur des Dolchesa und elitärer "Dikıatur des Säbels "gebrandmarkc. Die Unumgehbarkeir der Unterscheidung von Freund und Feind und der gervaltsamen Lösung des Klasscnkonflikts ist sine Erkennenis, die Kirchheirmer gleichermaßen in der "Theorie vom doppclten Fortschritc ' vermißr. Weil Lenin und Sorel den Kompromiß ablehnen und Freund und Feind zu uncerscheiden wissen, werden sie als revolutionäre Vorbilder gegen den Pazifismus der Sozialdemokratie benannt. Die bolschewistische Revolucionstheorie isc poirtisch, weil sie - auch dies genuin Schmitsche Theoreme - den auf eine Entscheidung drängenden Ausnahmezustand anerkenne und willens sei, den Klassenfeind in der ssouveränen Diktatur des Proletariats niederzuhalzen bis zur Verwirklichung sozialer Homogenität im sozialistischen Staat. ${ }^{10}$

Kirchheimer trägr diese Gedanken nicht als persönliche Glaubenssäıze vor. Und dech ist ein waches Interesse $3 n$ der militanten Kritik Lenins und Sorels nicht zu verkennen, ein Interesse, das sich mitunter auch von der Wucht dieser Kritik mitreißen läßr. Aus diesem Inceresse, das aus der Analyse mehr macht als eine bloße Diagnose, spricht jene Sympathie für eine akrive, kämpferische, entschcidungsfreudige Polirik, die für Kirchheimers politische Theorie und Verfassungstheorie so überaus bedeutsam isc.

Die Ausführungen mögen als erscer Beleg dienen für den Erkenntnisgervinn einer Rekonstruktion der Weimarer Arbeiten Kirchheimers im kritischen Vergleich mir der Theorie Carl Schmitrs. Ein weiteres Indiz für die sachliche Nähe der beiden Theorien ist im Wissenschaftsverständnis und im methodischen Vorgehen angezeigt. Einschränkend und zugleich bestärigend ist vorauszuschicken, daß Außerungen zum Wissenschafrsbegriff und zur angewandren Methode im Werke Schmirs rar sind und bei Kirchheimer fast vollständig fehlen. Die Vernachlässigung metho. discher und wissenschaftstheorerischer Fragestellungen wird erhellt von der Selbstverständlichkeit, mit der beide Staatsrechtler die enge Verwandischaft ihrer Wissenschaft zur Politik bejahen. Das Bestreiten dieser Verwanduschaft isc für Schmitt ein "policischer Kunstgriff", mit dem die eigene Position als unpolitische übcrhöht und die des Gegners als policisch zrveckoriencierte denunziert werden solle." Staats* rechtiche Theorien werden gewonnen in konkreten politischen Auseinanderseczungen mir der Folge, daß die wissenschaftliche Diskussion die Spannung des politischen Kampfes erhäls. Die Bezogenheic auf politische Situationen und Bewegungen untersagt ein Verständnis von Wissenschakr als merhodisches und systemarisierendes Arbeiren auf allgemeingülrige Erkenntnisse hin. Jede Erkenntnis ist Gegenwanserkennenis, jeder Geist nur gegenwärriger Geist, es gibr keine inrersubjektiv nachvollziehbare Erkenntnis, sondern nur Aussagen zur konkreten, immer in Streit befindlichen politischen Lage. Kirchheimers Wissenschaftsbegriff ist zuriefs: geprägr von dem seines Dokrorvacers. 1964 schreibt er in einem der ganz wenigen

8 Schmitt, FN 3i s. wuch die Aulsatzsammlung Schmilts . Donoso Conis in gesamieuropiischer Interpretstion:-, Köln 1950.

9 Donoso Cories. Rede vom 4. Januas 1849 über die politischen Grondsüıze ber Gegenvart und die Dikuaur, in: ders, Briele. parlamentarisehe Reden und diplomacische Beriehe aus den keizen Jahren seines Lebers (18+9-53). Hesg. A. Maier, Köln 1980, S. 199 If.

10 Kirchineimer, FN I, S. 45.

1) Schmik, Hugo PreuB - Sein Stazesbegrill und scine Secllung in der deutschen Stazslehre. Tübingen 1930.5 .6 . 
Särze, die sein Wissenschaftsverständnis thematisieren, es sei das "Handwerk " des policischen Analyrikers, « Regierungssysteme in voller Akrivitär zu dechiffrieren, zu diagnostizieren oder in seinem Geiste bessere tür sie zu substicuierenu. "2

Wie aber können aus den Kraftlinien des politischen Geschehens wenn schon richt - Wahrheiten-, so doch Evidenzen gewonnen werden? Woher bezieht die Sensibilität für politische Prozesse die Krafr, den Mur und das Selbscvertrauen, in einer "Zeit raschen Verschleißes politischer Systeme $\propto$ die Dynamik der Politik zu úberführea in stastsrechtliche Begrifflichkeit? Schmitt hat 1970 eine späte Antwort gegeben. wIch habe eine Methode, die mir eigentümlich ist: die Phänomene an mich herankommen zu lassen, abzuwarten und sozusagen vom Stoff her zu denken, nicht von vorgefaßten Kriterien. "' Was hier proklamiert wird, ist die Prioritäc des insuitiven Verstehens vor dem Geschäfr logifizierter Hyporhesen- und Begriffsbildung. Intuition hat ein innigeres Verhältnis zur Asthetik als zur Logik. Wenn eine gedankliche Struktur niche über "vorgefaßte Kriterien a zum racionalen Nachvollzug einlädr, wird sie ihre Wirkung in der Faszination des Prägnanten und Schönen erproben müssen. Soll ein Gedanke weinleuchten", wird er sich an den Anforderungen an ein Kunsrwerk hinsichtlich innerer Harmonie, Stimmigkeic und Plastizität zu messen haben. Der Stil wirkt als Eindringhilfe, die dem Text erleichtert, win die subjektiven Tiefen derer, die es angehr, vorzudringen und dort auf Wohivollen und Entgegenkommen zu treffenu. ${ }^{14}$ Bilder helfen beim Eindringen abstrakter Begriffe in die subjektiven Schichten des Verstehens. "Die an Schmitt bewunderte Formulierungskraft und Brillianz der Darsteltung bedient sich einer Prägnanz und eines Bilderreichrums, die den Leser überraschen und überwälrigen - oder aber ihn erschrecken und abstoßen. Auch Kirchheimers Schriften beronen den Aspekt des Stils und der Rhetorik, sie sind packend geschrieben, verzichten keineswegs auf ein Fortissimo starker Worte, sie sind agitatorisch. Denn die bezogenen Positionen sollen ja nicht im wissenschaftlichen Diskurs überprüft, sondern geworfen werden in die $*$ Waagschale der Zeit $\alpha^{16}$, in den politischen Kampt, wo sie sich zu bewähren haben. Die Arbeitsweise erfordert aul der subjekriven Seite des Analyrikers ein Gespür für politische Vorgänge, eine - wie Herz/Hula schreiben - ssensitivity to the historically relevant and the uniquely policicala."

Ein Ergebnis dieser Arbeitsweise liegt auf der Hand. Die sensitive Wahmehmung politischer Geschichte enzeugr Unstetigkeit im Interesse für Erkenntnisgegenstände und Sprunghaftigkeit bei der Rezeprion theoretischer Tradicionen. Das. Urteil von Herz/Hula . Orto Kirchheimer was nor a systemaric thínker $\alpha^{18}{ }^{\text {triff }}$ gleichermaßen Schmitts Werk, das jedoch kaum als * Werk $\propto$ im Sinne einer systemarisch encwickelten Theorie gelten kann. Die Form des wissenschaftlichen Arbeitens korrespondiert dem wissenschalestheoretischen und methodischen Selbscverstänonis. Uncer den Arbeiten Kirchheimers dominieren Aufsäıze, abgesehen von dem voluminösen Alterswerk solitische Justiz "' führ die von Luthardt erstellte Auswahlbibliogra-

12 Kirchheimer, Vorbemerkung zu der Autsarzsamulung: Polksk und Veriassung, Frankfurt am Main 1964, S. ?.

1) Sehmite in einem Gespräch mì J. Schickel, in: Sehickel, Guerilleros, Parisanen. Theoric und Praxis, Münehen 1970, S. 11

14 D. Sukr, Entialung der Mensehen durch die Menschen. Berlin 1976, S. 29.

is H. Triepel. Vom Seil des Rechus. Beieräge zu einer Astherik des Rechs, Heidelberg 1947, S. ${ }_{4} 6$ ff.

16 So Lutet eine gelungene Formulierung Schmitus in dem 1997 geschriebenen Vorwor zu: Verfassungsrecheliche Aulsäze aur den jahren 1924-1954. Malerialien zu einer Vertassungslehre, 2. Aufl., Berlin 1973.5 .8 .

1) La: Burin/Shell, Polities, Lw, and Sucial Change. Selected essays of Otro Kirchheimer, New York $1969,5 . x$

18 A. .. O.S. IX

19 Kirchheimer, Policische Jusxiz. Verwendung juristischer Veríahrensmöglichkeiten zu politischen Zwaken. Neuwied 1965 . 
phie ${ }^{10}$ kaum Bücher. Eine Bestandsautnahme der Arbeiten Schmitts ist vor einer analogen Einschätzung nicht schon dadurch gefeit, daß die von Tommissen erstellte Bibliographie aus dem Jahre 1958 in der Rubrik . Bücher und selbstandige Schriften 58 Tizel zähle." Denn bei näherem Hinsehen ergibr sich, daß ein gervichriger Teil der Publikationen Aufsaczsammiungen, kaum über Aufsarzformat hinausgelangte Broschüren oder in Buchtorm publizierce Vorträge sind." Den großen Scudien liegen viclfach Aufsätze, die vorab veröffentliche wurden, zugnunde." Aufsätze und Rezensionen dominieren ganz eindeurig auch in der Schmitr Bibliographie." Lie Form des Aufsazzes ist weiraus besser geeigner lür eine rasche Reaktion auf zeirgeschichtliche Vorgänge als die Buchform. Erschwert werden aber Syscematik und Kohärenz der wissenschafelichen Arbeit. Freilich muß der Mangel an Systematik nicht als defizitär begriffen werden, sprechen doch die Fülle der behandelten Themen und der Reichtum an Gedanken für die Produkrivitär der Arbeitsweise.

Trozz der wissenschaftstheoretischen und methodischen Ubereinstimmungen isc die Divergenz im Erkenntnisinteresse erheblich. Kirchheimer legr sein Erkenntnisziel offen, er will verantworklich mitarbeiten an der "Schalfung menschenwürdiger und sinnvoller gesellschaftlicher Zuständea." Vergeblich wird man in den Schriften Schmitus nach einer vergleichbaren Darlegung forschen. Die Nachbarschaft der Wissenschafc des öffentlichen Rechts zur Politik, auch zur Tagespolitik, wird zwar ständig betont, dennoch insistien Schmitc auf der politischen Neutralität des Wissenschafters. Das Mißverhältnis zwischen einem politischen Wissenschafrsverständris und der bcharrlichen Verteidigung einer über die Politik erhabenen Integritä̌ der Person des Wissenschafters wird auf die Spitze getrieben im Begriff des "Schicksalsa. Der Gedankengang ist folgender: Die Uberschneidung von Wissenschaft und Policik ist ein sachlich fesizuhalcendes Fakrum; der Wissenschaftler, der dieses Spannungsfeld bearbeiter, wird wider seinen Willen hineingezogen in den Malstrom der Politik. ${ }^{25}$ Die Deutung solcher Aussagen als Apologie der im -Dritren Reich « eingenommenen Rolle ist noch niche einmal die halbe Wahrheit. Als der Fraktionsführer des Zentrums, Prälat Kaas, am 26. I. 1933 in einem Brief an die Reichsregierung vor den ndas gesamte Staatsrecht relativierenden Grundtendenzen von Karl (!) Schmit und seinen Gefolgsmännern “ wamte", reagierte Schmiı verärgert und beleidigt. Sein Antwortbrief vorn r. 2. $1933^{28}$ enthält zwei Argumentationsscränge. Er, Schmitr, sei in wissenschafrlicher Redlichkeit eben zu anderen Ergebnissen als andere Staassrechcler gelangr; es sei wUnrechra, aufgrund abweichender Ansichren die srecheliche Gesinnung e eines Rechrsgelehrten *politisch zu verdächtigen«. Um was Schmitc kämpfr, ist die Erhaltung eines Restes von Person: der niche aufgeht in politischer Absicht und der generell unverfügbar bleiben soll

20 In: Kirchibeimer, FN I, S. 246 If.

11 In: Festschrift für Carl Schmist zum 70. Geburstag, hesg. von Barion, Forstholf, Weber, Berlin 1959, S. 27$\}$ If.

is Die Bibliographie iühr in der genannten Rubrik s Aufsaczsammlungen und is Brosehüren mir weniget als so Seiten. Zieht man davan noch 3 Vorträge, die niche sehon unter - Broschüren- in Anschiag gebrachr sind, und die ${ }_{4} 4$ Zweil- und Mehraufagen ab, verblciben 2 Rublikationen in Buchform.

2) Hierzu gehören die Bücher: Die Dikrzrur. Die geislesgeschichtliche Lage des heucigen Parlamenrapis. mus, Der Begriff des Polizischen. Der Hürer der Verfassung und - bedingt - Der Nomas der Erde. Köln 1950.

24. Die zilierte Tommissen Bibliographie zähle annahernd 200 Aulsätze.

15 Xirchheimer, FN 12, 5. 7

26 Schmite, F.x Caprivirate Salus, Köln s950, S. s6

:7 Verölíenelicht in: Jahrbuch d. öllentl. Rechts, Bd, 2r, S. 14t.

28 Erstmals verólfentlicht in: P. Tommissen, Over en in Zake Carl Schmict, in: Eclectica, s de Jaargang Nr. 2, Brussel, S. 111 i. 
Die existentialistische Fassung und Immunisierung wissenschafelicher Verantwor-

tung fordert geradezu heraus, das Ureil über das rvissenschaftliche Werk zu verbinden mit moralischen Einschäczungen der Person.

Aus leicht einsehbaren Gründen arurde bisher der Einfluß Schmitts auf die Theorie Kirchheimers hervorgehoben. Als Kirchheimer im Alter von 23 Jahren seine Dissertation schrieb, war der qojährige Schmit bereits einer der angesehensten deutschen Staatsrechilicher. Gelernc hat aber auch Schmitr von dem jungen Promovenden, was schon die häufigen Zitierungen belegen. ${ }^{19}$ Bei der Lekrüre der Zitace drängr sich der Eindruck auf, daß Kirchheimer Anregungen aus der manxistischen Diskussion, zu der Schmitr den Kontakt nicht abreißen lassen wollte, vermitreite. ${ }^{\circ 0}$ Uber die persönlichen Beziehungen der beiden ist mir nichts bekannt.' Daß Kirchheimer von seinem Doktorvater beeindruckt war, läßt sich aus der Rezeption Schmitcscher Theoreme unschwer ablesen. Im Sommersemester 193 i hatre Kirchheimer gemeinsam mir F. Neumann ein verfassungsrechcliches Seminar bei Schmict an der Handels-Hochschule in Berlin besuchr. Der ungewöhndiche Vorgang, daß ein deutscher Staatsrechesprofessor in z wei wissenschaftlichen Aufsä:zen sogar Kirchheimers Beiträge zur Seminardiskussion zicierr, könnte auf ein fast freundschaftliches Verhälınis hinweisen. ${ }^{12}$ Verstärkt wird die Annahme von dem Tarbestand, daß Schmitr und Kirchheimer Manuskripte ihrer rvissenschaftichen Arbeit vor deren Publikation ausgerauscht hatren."s

\section{Verfassung und Entscheidung}

Die 1930 erschienene Schrift $*$ Weimar - und was dann? ${ }^{33}$ kann zu den klassischen Analysen der Weimarer Reichsverfassung gerechnet werden. Das Faszinosum, das von der in der wongsozialistischen Schriftenreihe" publizierten Studie ausgeht,

xq Vgl. FN 9. Weitere Zitierungen finden sich in den Aufsäвen aus FN I6: Das Reichsgeriehe als Hüter der Verfassung (1929), S. 99: Freiheierechee und insprucionelle Garantien (1931), S. 161/2, 168; Grundrechce und Grundpflichuen (1931), 5. 182, 192, 195; Legalirät und Legicimitäı (1932), S. 169. In den Nachbemerkungen zu den Aulsäczen nimmi Sehmitr igs7 u. 2. auch Bezug uuf nevere Arbeiken Kirchheimers, vgl. S. $262,346,366,488$.

30 Dafur sprechen die Zitate S. 162 und 167 , Hinweis auf K. Reancr, Die Rechusinsuitute des Privatreches und ihre soziale Funktion. Tübingen 1929, und der Kontexe des Zitats FN s: Bauer, Kaucsky, Adler, Gurland.

31 Rainer Erd hat in einen Gespräch mir Carl Schumire in Juli 1980 den wertvollen Hinweis erbalten, daß Kirchheimer ihn noch in dea funfziger Jahren in Pletrenberg besuche hacee.

Schmiu sehricb hierzu an j0. 9. 1968 in einem Brie! an J. Seifert: - Meine N2ehkeiegsbeziehungen zu Herm Kirehheimer beginnen mil seirem Besueh in Plenenbers (2m 27. November 1949) und endecen im Sommer 1961, als ich Einzelheiten über sein Verhalren in dem Promocionsverdahren Sehwab effuhr. . . Kirchheimer ha dic Annahme der Arbeir as einc An und Weise verhinden, die mich cinen seit 1927 von mir genähren Irrum erkennen ließ.- Aus einer sehr persönlichen Perspeksive berichret Sihwab viber das Verfahren und das Rigorosum bei Kirchheimer in dem Aufsaz: Through a Glass Darkly, in: Canadian Joumal of Political and Social Theory, Vol. 4, No. 2. (Spring Summer 1980), 149 ff. Frau Gurland hxe mir in eirem Gespräch milgeteilt, Kinchheimer habe das Promotionsverlahren als cine hineeryruinde Strategie Schmutus geweret, scine Rehabilitseion via USA und unter Bcnutzung der Person Kuechheimers zu betreiben; zu dioer Surategie habe er, Kirchheimes, seinc Hand niclie reichen wollen.

32 - Die in meinem verlassungsthearerischen Seminar Sommer 1951 von O. Kirchheimer formuliene -allgemeine These, daB überhauge nur cin justizstaat . . Grundrechte haben könne, soll hier wenigatens erwähne serden. FN 16. S. 192, ahnlich S. 168.

3) So zitien Kirehheimer aus dem -unveröffenulichen Gurachen. Schmius zum deuesch - polnischen Abkommen vom 3t. Okcober 1919 (publizier unter dem Titel: Racilikation völkerrechnlicher Verräge und innersezatliche Auswirkungen der Wahrnchmung suswäriger Gewale, wiedersbgedr. FN 16, S. 11+ff.) un seiner Arbeic: Die Grenzen der Enreignung (1930), wiederabgedr. in: ders., Funkrionen

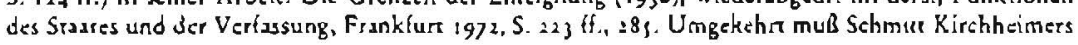


gründet in der gelungenen Verbinulung von soziologischer Realanalyse und Verlassungscheorie. Lorenz von Secin" und Karl Marx sind unschwer als Vorbilder dieses Ansazzes auszumachen. Marx hatec den "umbassenden Widerspruch in der tranzösischen Konscirucion vom 23.10.1848 damit erklärt, daß derr vierten Stand mit dem aligemeinen Stimmrecht der Zugang zur politischen Macht eröffner und zugleich die soziale Machc des Bürgertums verfassungsmäßig garantier sei.." Ahnlich wie in seiner Dissercation rückt Kirchleimer auch hier die Annahme, ein Gleichgewicht $z$ wischen den sozialen Klassen verbürge die Lebensfähigkeit einer Verfassungsordnung, in das Zenrrum seiner Kritik. Entgegengesetze zur Position Anschürzs, der den verfassungsgeserzlich verankerten Klassenkompromiß als Stärke der Verfassung feiert," erblicke Kirchheimer in der Kompromißstrukrur das politische Dilemma der Reichsverfassung. Denn die Reichsverfassung sei kein echter Kompromiß, der positiv definiert isc als gegenseitiges Nachgeben zur Erreichung einer daverhaften und eindeutigen Regelung, vielmehr sei das vorgeblich Kompromißhafte eine win der Verlassungsgeschichte bisher unbekannte, einzigartige Nebeneinanderordnung und Anerkennung der verschiedensten Wersysteme *. Das Fehlen einer Entscheidung für ein inhaltliches Organisationsprinzip degradiere die "Verfassung ohne Entscheidung "zu einer "formalen Spieiregel $*$, über die der sozial Mächtigere im Kräfteparallelogramm beliebig verfügen könne.

Bis hierher reiche die Analyse kaum hinaus über die Tradierung marxscher Positionen aus den politischen Schriften, angereichert mit Fragmenten der Schmittscher Verfassungslehre und Demokracietheorie. ${ }^{88}$ Die Gedanken zum Wechselverhälınis von Legalstruktur und sozialer Mächrigkeit entfalten hingegen eine erstaunliche diagnostische und prognostische Qualität. Die Machesäulen im politischen System von Weimar, vorrangig Reichswehr und Bürokratie, widerlegen drastisch die Einschätzungen der WRV als offener Verfassung. Beeindruckend ist Kirchheimers treffsichere Analyse von Art. 48 WRV. Der Diktaturartikel eröffne die legalisierte Chance, die Alternative pariamentarische Demokrarie oder Diktatur zu bchandeln als «Zweckmäßigkeitsfrage, die nur unter dem einen Gesichtspunkt zu entscheiden ist: was dient am besten der Aufrechterhaltung des ökonomischen status quo? „' Die Kriterien jener Zweckmäßigkeit werden von Kirchheimer encwickeir im Beziehungsfeld dor „Direkrionssphäre*, die der Gescrzlichkeir des kapitalistischen Produkrionsprozesses vollkommen unterworfen ist, und der *Verreilungssphäre , in der die politischen Kämpfe um die Verteilung des Sozialprodukts ausgetragen werden. Entgegen der Kritik einiger Autoren der ${ }_{n} N e v e n$ Linken ${ }^{* 0}$ ist festzuhalten, daß die Differenzienng von Direkrions- und Verreilungssphäre nicht eingeführt wird, um die gewerkschafclich-sozialdemokratische Politik der Verteilungstämpfe zu legicimieren, sondern im Gegenteil soll das

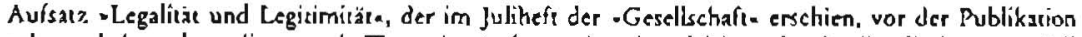
gekanne haben, da er die zentrale These des Aufsarzes in seine gleichnarnige Srudie, die ja am 10. Juli $19\} 2$ abgeschlossen vorgelegen haben soll, eingearbeiter hate. Vgl. FN 16, S. 26$\}$ f6, 269. Zovar nennt Schmin die Fundsrelle des Aulsatzes, alicrdings ohne Seirenangabe, woraus zu sebließen ist, daB ihm der Erscheinungsort von Kirchheimer vorab migeteilt worden war.

34 Wiederabgede. in: Kirchheimer, FN 12, S. 9 ill.

us $\mathrm{Vgl}$. die Fußnore 24 , 2. 3. O.. S. 1ss.

16 K. Marx, Die Klasscnkämpfe in Frankreich 1848 bis 1850, MEW, Bu. 7. S. 9 If., 43; hierzu Kiechheimcr, 3, 2. O., S. 16.

37 G. Anschürz, Drei Leirgedanken der Weimarer Reichsverfassung. Tübingen 1921, S. 26.

$38 \mathrm{Hern} / \mathrm{Hula}$, FN 17, S. X, urcilen sbenso: ... a peculiat amaigamacion of Schmarism and Marxism.

19 Xirchheimer, 1, 2, O., S. 20

$40 \mathrm{Müiler/Ncusuß,} \mathrm{Dic} \mathrm{Sozialstaassillusion} \mathrm{und} \mathrm{der} \mathrm{Widerspruch} \mathrm{von} \mathrm{Lohnarbett} \mathrm{und} \mathrm{Kapital,} \mathrm{in:} \mathrm{Trokla.}$ Sonderheft I, (19;1), S. Iy und 49, werien Kiechhcimet vor, er sci mus der Trennung con Dirckrionsund Vereilcrsphare zum Vorreier modemer Sozialseratsglaubigkes geworden. 
aufgezeigte Mißverhälınis zwischen polirischer Mechanik und ökonomischer Gewalı allen Illusionen hinsichelich der Autonomic der Verteilungssphäre entgegenwirken."

Eine whochinteressante Schriftu hat Schmitr diese Analyse genannt und ihren Ergebnissen -durchaus zugestimmr a." Das Urreil verwundert nicht, denn analoge Ausführungen zum Weimarer Verfassungskompromiß stehen bis in einzelne Formulierungen hisein in der "Verlassungslehre a aus dem Jahre 1928. Einverstanden kann Schmir sich zeigen mit dem verfassungscheoretischen Ansatz, soweit er positiv orientiert ist am Entwurf einer Verfassung als inhalclicher Entscheidung. Die pauschale Zuscimmung läßt jedoch die Divergenzen außer aclst. Nicht übemommen har Kirchheiner die für den positiven Verfassungsbegriff konstitutive Unterscheidung von Verfassung und Verfassungsgesetz. Nur den mbloßen * Verfassungsgeserzen vornehmlich des Grundrechesteils mic seinen sozialen Verbürgungen wird in der *Verfassungslehre* das Fehlen des Entscheidungsmoments zugeschrieben, wohingegen der pouvoir constiruant im organisatorischen Teil eine posizive Entscheidung über Form und Arr der politischen Einheil gefäll habe, nämlich entschieden habe zugunsten des wliberalen Rechesstaates mit demokracischer Staatsform * und gegen die $\times$ Räterepublik mit Dikcatur des Proletariatsa." Verfassungstheoretisch gesehen ist die Divergenz zu Kirchheimer trorz der beiderseitigen positiven Unterstreichung des Entscheidungselements erheblich, unter dern Aspekr der soziologischen Realanalyse becrachret aber äußerst gering. Denn die sozialen Inhale, die Schmicts verfassungstheorezischer Dezisionismus in der "Subsranz des positiven Verfassungsbegrifts unter eine Evigkeitsgarantie stelit, sind identisch mit den Ergebnissen jener heimlichen Entscheidung für den gesellschaftlichen status quo, die Kirchheimers sozialwissenschafelicher Ansatz herausschält. Was dieser als Jemokraciewidrige Anomalie beschreibt, hat Schmict nur verfassungstheorerisch verpackt: „Die Entscheidung mußre für den bisherigen sozialen status quo, d. h. hür die Beibehaltung der bürgerlichen Gesellschaftsordnung fallen . . . at4 Gewiß liegen der Differenz von Dezisionismus und sozialwissenschaftlicher Analyse antagonisrische verfassungspolitische Oprionen zugrunde. Schmitr sichert die Verfassungsordnung ab gegen Entwicklungen in Richtung Sozialismus und häl alle anderen Opeionen offen, während Kirchheimer in einer konsequent sozialiscischen Policik, die alie den status quo repräsentierenden sozialen Machtballungen demokratisch bändigt, die einzige Uberlebenschance der Verfassung erblickr.

Wie kann die deutsche Arbeicerbewegung auf dem Boden der Weimarer Verfassungsordnung wieder politisches Terrain gewinnen? Die Frage ist das Leirmociv der Schriften Kirchheimers. Der Sozialdemokratie wird zunächst die Einsiche abverlangt, daß die bloße Verteidigung des Erreichten, das zähe Festhalten an den einmal errungenen Positionen eine bedenkliche Selbsibeschränkung ist angesichrs einer stürmisch vorpreschenden Kontertevolution: „Es gibt nur ein Vorwärs oder ein Rückwärts*." Kirchheimer will keineswegs die sozjalistische Revolurion auf die Tagesordnung serzen, er geißelt vielmehr eine solche Politik an der KPD als $»$ Takrik des Pursches ${ }^{\circ 6}$ Zwischen dem heute und dem morgen gibt es keine Grenzen, dieser Satz wird den sozialdemokrarischen Realpoiicikem ebenso in das Stammbuch geschrieben wie den von dem großen Bruch trüumenden Revolutionsenthusiasten

t1 Kirchheimer, 2. 1. O., S. +2 If.

+2 Schmise. FN 29. S. 192

4) Ders. Verfassungslehre, 5. Aul!., Berlin 9970. 5. 36.

it A. 2. O., S. 31.

as Kirchhesmer, Verfassungswirklichkeil und politische Zukunir der Arbeirerklasse, FN I, S. 69 ff., 76. +6 A. . . O. S. 73 . 
der KPD. In den Kämpfen von heute muß das Morgen enthaiten sein, eine linke Politik muß den Massen sozialistische Ziele, eine konkrete Uropie angeben können."

\section{Legalität oder Legitimität?}

Franz Neumann schrieb 1930 zur Schrift seines späteren Freundes Kirchhcimer, die Analyse der Weimarer Verfassung komme sehr stark kommunistischen Ideengängen* nahe. ${ }^{8}$ Das nicht näher begründere Urteil hat eine gewisse Seütze in der revolutionären Begeisterung, die aus den Schriften der Jahre 1928/29 und bedinge des Jahres 1930 sprichr. Die Euphorie der Entscheidung tendiert auch zur Vereinfachung sozialer Sachverhalte, wofür die grandiose Fehleinschätzung der "Deurschen Demokratischen Partei* ein anschauliches Beispiel ist." Trotz der kämpferischen Linie sind selbst die ersten Arbeiten Kirchheimers recht weit entfernt von den Positionen einer KPD, die für die Gleichgültigkeit der Geschichte gegenüber ihren revolutionären Deklamationen die ssozialfaschistische sPD verantwortich machte. Kirchheimers Blick ist nach vorn gerichtet, das Gerede vom -Verrata der SPD bringt die Dinge nicht voran, die Sozialdemokratie muß aufgerütrelt werden aus ihrer politischen Lethargie. Aber nicht nur politische Weitsicht und höherer Realitärsbezug kennzeichnen den Uncerschied zum politischen Selbstverständnis der KPD. Gerade in den Teil der Analysen, die KPDEinschätzungen am nächsten zu kommen scheinen, nämlich im Nachweis der Instrumentalisierbarkeit der Reichsverfassung aufgrund ihrer Unentschiedenheit, ist auch die Differenz am sichtbarsten. Für Kirchheimer ist die Verfassung ein gesellschaftliches Organisationsprinzip, dessen Mängel im politischen Kampt zu beseitigen sind. Verfassungsfragen sind zwar stets auch politische Fragen, jedoch verbietet der Formalitätsvorsprung des geltenden Verfassungsrechts die Bagatellisierung der Verfassung als xideologisches Úberbauphänornen a und ihre Verflüssigung in den Stoff politischer Alltagstaktiken. In der Behandlung materialer verfassungsrechtlicher Streitragen ist schon 1930 eine Verschiebung der verfassungstheoretischen Betrachtung weg von der These der. Unentşchiedenheit und hin zur Akzeptanz einer unzweifelhaften Geltung einzelner positiver Verfassungsgesetze festzustellen. ${ }^{10}$

Der tiefe Einschnitt, den der Rücktritt der letzten parlamentarisch getragenen Reichsregierung am 30. März 1930 in der Geschichte der Weimarer Republik markiert, bleibr nicht ohne Wirkungen auf die Theorie Kirchheimers. Ein Aufsatz zum Verfassungstag 1930 stellt die Weichen tür eine vorsichtige Umorientienung." Kirchheimer nückt ab von der Kritik an dem unechten, die Entscheidung vertagenden Kompromiß und charakterisiert nun die Zusammenarbeit von Sozialdemokratie und Tcilen des Bürgertums als Grundbedingung wowenigstens ein Stück Demokracie». Diesen gemeinsamen Boden habe das Bürgertum gefahrlos verlassen können,

47 A. . . O., 5. 76

8 Neumann, Die soziale Bedeutung der Gnundrechec in der Weimarer Verfassung, in: ders., Winschak, Sraar, Demakratie. Aulsärze 1930-1934. Frankfurt am Main 1978. S. 74.

49 Die Demokextische Parei sei - wegen ihrer Ideologie der Arbeiterschafe weir gefährlicher 1 is deren oflene Feinde., leider sei dies - leitender Pareikreisen. noch niche klar geworden. Kirchheimer, Bedeuningswandel des Parlamentarismus (1928), FN I, S. j8 81 .

so Vgl. die Sudie: Grenzen der Fnreignung. FN 3j. S. 223 ff., hice 25 iff.

sI Ders.. Arrikel 48 und die Wandlungen des Veriassungssystems. Auch ein Beierag zum Veríassungstag (1930), FN I, S, 91 ff. 
da Ar. 48 WRV den Rückgriff auf Herrschaftsmittel gegen parlamentarische Mehrheisen erleichtert habe. Die Zukunft der Republik ist nunmehr untrennbar verbunden mit der policischen Strategie der Sozinldemokracie: "Die Sozialdemokralie... wird ihren Anhängern gegenüber auch keinen Zweifel darüber aufkommen lassen dürlen, Jaß die Zeir der Kompromisse vorüber ist und die Zeir der staatserhaltenden Selbsierhaltung begonnen har. "s"

Die Bestimmung des Verhältnisses von Verfassungsrecht und -politik ist das Kirchheimer und Schmirr gemeinsame Anliegen der Folgezeit. Das Begriffspaar "Legalität und Legitimität bilder die kategoriale Siruktur, in der das Thema verorter ist." Für Kirchheimer ist die politische Ordnung der parlamentarischen Demokracie legicim, wenn alle Beschlüsse den Anforderungen legaler Verfahren genügen und zurückführbar sind auf das demokracische Mehrheicsprinzip. Legalität und Legitimität fallen zusammen unter der Bedingung gesetzmäßiger Entstehung und Ausübung von Macht. Das Nocverordnungsrechc nach Axt. $4^{8}$ WRV aber durchbreche die scharfe Trennung von geserzgebender und regierender Gewalt und setze sich so dem Zwang aus, außerhalb des Legalitätssystems eine neue Legitimitätsgrundlage zu finden. Die Berufung auf eine indiskutable materiale Richtigkeit der Handlungen und Ziele bürokratischer Herrschaft sind gemeinsam mit einer prätendierten Unparteilichkeir und Neutralität die Elemente der neuen Legitimität. Kirchheimer weist nach, daß die soziale Basis der Präsidialkabinette, Staarsbürokratie und Reichswehr, zu schmal ist, als daß sie real die prätendierte Rolle des höheren Dritcen zwischen den rivalisierenden Parteien des Wirtschattslebens zu tragen vermöchre. Die Präsidialdikratur müsse deshalb Anlehnung bei jenen sozioökonomischen Kräfren, die mit ihrem konservativen Sozialideal übereinstimmen, suchen.

Die Bescandsaufrahme Kirchheimers impliziert die Warnung vor den Folgen des Experiments, in die Verfassungsordnung von Weimar eine überlegale Legitimitätsebene einzuziehen. Dagegen lassen sich Schmitrs Arbeiten seit dem Jahre 1930 interprevieren als mit wechselnden Argumenten und zunehmender Intensität vorgetragenes Plädoyer für die Inscallierung der Präsidialkabinette. Im „Hüter der Verfassung sind die Bausteine für jene Theorie des starken Staates gelegt, die den Autoren bis heure mit einem zweideutigen Benühmtheitsgrad ausgestatter hat. Zwei Strukturprinzipien kennzeichnen den kritisierten parlamentarischen Geserzgebungsstaat der ausgehenden zwanziger Jahre: die Herausbildung des Wirtschafusstaates und des pluralistischen Parteienstaates. Zwar profitiert der aus Schwäche rotale Staat des Pluralismus und der Polykracie von den Intervenrionsmechanismen des Wirschaftsstaates, jedoch bestehr zwischen beiden Staarsformen keine strukturell notwendige Verbindung. Desha'b wirò die Strategie möglich, einerseits die Mächte des Pareienstaates zurückzudrängen und anderseits den Wirtschaftsstaat zu stärken mittels der Uberantwortung seiner Interventionsmöglichkeiten an eine entscheidungstähige, sachlich neutrale Elite."Schmitr stellt die Republik vor die Encscheidung zwischen Legicimität oder Legalicät, zwischen *Anerkennung substanzhafter Inhate und Krätce des deutschen Volkes“ oder . Bcibehaltung und Weiterführung der funktionalistischen Wercneurralität mir der Fiktion gleicher Chance für unterschiedslos alle Inhalce, Ziele und Strömungen“."Der konstatierte

(2 A. 1. O.. S. 95.

13 S.hnnit, Legalitat und Legitimitat (1932), in: F.V 16. S. 263 f., Kirchhcimer, Logalitże und Legitimitear (iyj2). in: Politisishe Heerschalt. Fünf Bcitrige zur Lehre vom Sesat. Franklure 1967, S. 7 if.

SA - Enrparcipulitisierung. heißst Jic von Schmint. FN , S. 110 , vorgeschlsgene Abhilfe gegen die Kräfre des kialitionsparteiensitades.

is Ders., FN \&3. S. 3.4. 
Zusammenbruch des Lcgalizärssystems antizipiert die Entscheidung für die vom Reichspräsidenter über Art. 48 WRV zu wahrenden "substanzhaften Inhakre، des zuvor von sozialen Verbürgungen und politischen Freiheissrechten gereinigten Grundrechtsteils ${ }^{6}{ }^{6}$ und gegen die rvertneutrale Legalität des parlamentarisch-demokratischen Gescrzgebungsstaates.

Kirchheimers gemeinsam mit Nathan Leites verfaßte Replik wurde leider erst im Jahre 1933 publiziert, also zu späc, um noch politische Wirksamkeir entfalren zu können." Uberaus deurlich wird in dieser Schrifr die Emanzipation von den Lehren des Doktorvaters. Die Verfasser bestreiten das Grundtheorem der Schmietschen Demokratielehre, nämlich die Loslösung des demokrarischen Gleichheitspostulats von einem rechusstaatich gefaßcen Freiheitsbegriff. Die Verengung des Freiheirsbegriffs auf eine staatsfreie private Sphäre des Individuums müsse den Bezug von Freiheit auf öffentliche dernokratische Willensbildungsprozesse aus dem Blickfeld des Ansatzes drängen mit der beklemmenden Folge, da $B$ in den Gleichheitsbegriff die Willensemanationen freier Individuen als prinzipiell gleiche nicht mehr eingehen können. Gleichheic kann nun umgeschmolzen werden in jene Gleichartigkeiten, wie Nation, Rasse, Religion erc., die jede Verbindung mic dem demokratischen Urprinzip niMehrheit entscheidet abstreifen. Demgegenüber insistieren Kirchheimer/Leites auf der ideen- und realgeschichtlichen Koppelung von Freiheit und Gieichheit als demokracischer Strukturprinzipien. Die Demokratie lebt nicht von der Existenz einer bestehenden Wertgerneinschaft, sondern stelit einen Funkcionsmodus bereic, mittels dessen die sozial heterogenen Gruppen einer pluralistischen Gesellschaft ihre jerweiligen Wertvorstellungen verwirklichen können.

Kirchheimer ist zu sehr politischer Analyriker, als daß er bei der Idealkonstruktion einer formalen Demokratie in einer pluralistischen Geseilschaft stehen bleiben könnte. Denn was geschieht, wenn einer Gruppe die außerlegalen Machrchancen größer erscheinen als die legalen? Kirchheimer gibr eine verfassungsthcoretische und eine polirische Antwort. Gerade die von Schmitt als Zerfallserscheinungen des Legalitätssystems bezeichneten materiellrechtlichen Sicherungen der Reichsverfassung könnten in dieser Situation stabilisierend wirken, indem sie bestimmre Sachgebiere dem Zugriff einfacher Mehrheiten entziehen und diejenige soziale Gruppe, in deren Interesse solche Fixierungsnormen bestehen, zu einem positiven Verhältnis zur Demokratie motivieren. Ähnliches gilt für jene Verfassungsbestimmungen, die lediglich Programmsätze und Ermächrigungsnormen sind. Sie versprechen der Parei, deren Programmatik mit dern Inhalt jener Normen übereinstimmt, die Legalität der Machterweitenung, d. h. sie wirken verfassungspädagogisch und integrierend. Trotz der Integrationskraft des positiven Verfassungsrechts aber ist es politisch immer möglich, daß eine Machogruppe ihre Zielsetzungen mir außerlegalen Mitreln zu realisieren crachrec. Ist diese Gruppe mächtig genug, ihre Programmatik außerlegal durchzuseızen, müssen legale Gegenminel versagen, über das Schicksal der Demokratie entscheidet nun der politische Kampf. Noch sichtbarer fällt der Bruch mit den von Schmirt und ihm selbst bis 1930 verretenen Lehren in der Einschätzung des Parlamentarismus aus. Das Parlament wird begriffen als -piebiszitäre Zwischenschaltung* und "demokratisches Transformationsorgan* im Entscheidungsprozeß der Demokratie. ${ }^{8}$ Es verbürgr eine effekrive Mehrheitsermittlung, nachdem Mechanismen unmittelbarer Demokratie in den modernen Massengesellschaften fragwirdig geworden sind.

36 A. a. O.S. 345 .

37 Kirchheimer/Laires, Bemerkungen zu Carl Schmitrs -Legalieär und Legitimirase(1913). FN I. S. ilifl.

s8 A. 2. O..5. iss. 
Die Gründe für diese Revision der eigenen Position sind zuerst zu suchen in einem Wandel des marxistischen Selbstversiändnisses. In der letzren der Weimarer Periode zuzurechnenden Publikation's zweifelr Kirchheimer, ob die der Herrschaft des Proletariars vorausgehende Srazrsform norwendig die bürgerliche Demokratie sein müsse. Die Erschürterung des marxiscischen Glaubens an eine Geserzlichkeit im historischen Geschehen ist verursache durch die Herausforderung des Faschismus, der die Annahme einer geserzmäßigen Entwickhing zum Sozialismus drassisch widerlegt. Das Problem der Demokratie muß sich der Arbeiterbewegung nun in zweifacher Weise neu stellen. Zum cinen wird die Erhaltung der Emanzipationschancen der bürgerlichen Demokratie zum Kampfauftrag der Arbeiterklasse. Anderseits zwingt die faschistische Offensive zu einer Neubestimmung der Kamplmittel. Die Verceidigung der Legalirär darf sich nichı im blinden Glauben an still wirkenden Kräfre der Legalordnung erschöpfen, vielmehr muß die Arbeiterbewegung vorbereiter sein, die Staatsmachr in dem ihr aulgezarungenen Bürgerkrieg zu erobern. Das Handlungskonzept, das - projizier auf die Policik der beiden Arbeiterparteien - die Alternstive einer reformistischen und einer revolutionären Strategie verklammert, ist das Produkt der Verarbeicung zweier verfassungspolitischer Konflikete, die drei Jahre der Agonie der Republik prägten.

\section{Die Verfassungsreformdiskussion}

In der Endphase von Weimar ließ die Spannung zwischen der Legalordnung und der Machrausübung den Gedanken verführerisch erscheinen, mitrels einer Verfassungsreform die Diskrepanz zugunsten der catsächlichen Herrschafrspraxis zu beseitigen. Die im *Herrencluba geschmiedeten Pläne eines NNeven Staates zielten auf eine Entmachtung des Reichstages und die Stärkung der Regierung, auf eine "machtvolle und überparceiliche Staaisgewaltu, die über den gesellschaftlichen Kräfren stehen solle wie ein wrocher de bronze ${ }^{60}$ Auch die zahireichen Reformvorschläge deutscher Staatsrechtier liegen nahezu einmütig auf der Linie eines Ausbaus der Befugnisse der Exekurive und einer Einschränkung der parlamentarischen Verantwortlichkeic der Regierung. ${ }^{\text {.1 }}$

Ein wichtiger Aspeks der Verfassungsreformdiskussion ist die Beteiligung von Juristen, die der Sozialdemokratie nahestanden. Ernst Fraenkel schrieb in einem vielbeachrecen Aufsatz, wer die Aufrechterhaltung der Verfassung wolle, müsse ihre Reform bejahen, andernfalls werde der Grundgedanke der Vefassung auf illegalem Wege beseitigt. Das Faktum, daß die Machrposicion der Exekurive bis zu dem *gegenwärtigen Zustand einer faktischen Diktatur a"ausgebaur worden war, wird nicht erklär aus dem Machtwillen der hinter dem Reichspräsidenten stehenden sozialen Kräfte, sondem mit der Unfähigkeit des Reichstages, die ihm verfassungsgeserzlich zugewiesenen Aufgaben zu bewältigen. Die Funktionsunfähigkeit der Legalordnung gebiete der SPD, sich aus ihrem * Vesfassungsfetischismuse zu lösen, Weimarer Reichsverfassung und Sozialdemokratie seien keine ssiamesischen Zwil-

59 Kinhtheimer, Marxismus, Diktacur und Organisacionsform des Prolecariats (1933), in: ders., FN 33, S. 100 il.

60 Papen in einer Rede vom 12. Okiober 1932, ziticr nach: $K$. D. Bracher, Dic Aunlösung der Weimarer Republik. 2. Hufl., Stukgars igs7. S. St4.

6r Vigl. die Beitrage in: F. Berber (Hrsg.). Zum Neubau der Verfussung. Berlin 1913.

6. Vcrissiungsrciurm und Sozialdemokratic, wiederabgedr. in: ders. Zur Soziologie der klasserjusciz und dufsïze zur Veríssungskrisc 19j1-32. Darmseadt 1963, S. sو ff., 90. 
lingea. Zur Wiederherstellung der Kongruenz zwischen Legałordnung und Machrausübung wird vorgeschlagen, in Art. $\$ 4$ WRV ein konscrukcives Mißcrauensvorum einzubauen und das Aufhebungsverlangen von Norverordnungen durch den Reichstag (Art. $4^{8}$ Abs. 3 WRV) unter den Vorbehalt eines vom Reichspräsidenten einzuleitenden Voiksentscheides zu steilen. ${ }^{6}$

Entgegen den Autfassungen Fraenkels und anderer sozialdemokratischer Autoren ${ }^{6_{4}}$ bestrcitet Kirchheimer, daß es den Befürwortern der Verfassungsteform um Papen lediglich um cire Neugewichcung der Machtverteilung zwischen zwei Verfassungs. organen als prinzipiell gleichberechtigter Legalitätsfaktoren gehe. Fraenkel habe einer funktionierenden Verfassung einen Eigenwert eingeräumt, ohne zu fragen, wanum die geltende Verfassung nicht funkvioniere und in welchem Interesse eine reformierte Verfassung funktionieren wïrde. "Für Kirchheimer ist es ausgemacht, daß die Verfassungsrcformplänc der Regierung Papen - Gayl zum primären Ziel haben, die politische und soziale Machrstellung einer begrenzten Bevölkerungsschicht, nämlich der höheren Bürokratie, des Großgrundbesitzes und der Industrie nebst ihrer sozialen Annexe, verfassungsrechtlich zu scärken. ${ }^{66} \mathrm{Die}$ Analyse klingt soweit plausibel, sie gibt aber noch keine Antwort auf die drängende Frage, wie die Sozialdemokratie gegen die reale Selbstentmachtung des Reichstages und das Leerlaufen der verfassungsmäßigen Machtverteilungsmechanismen agieren könne. Die gegen die Aushöhlung der demokracischen Verfassung konzipierte Strategie gehört zu den originellsten Analysen Kirchheimers. Die real vorhandene Spannung zwischen Legalordnung und Machtausübung könne in zweifacher Weise ausgeglichen werden: entweder durch die Anpassung der Legaistruktur an eine demokratisch regredierte Realität, oder durch deine Umgestaltung der sozialen Verhälınisse, so daß eine sinnvolle Ausfüllung der Legalordnung erreicht werde. ${ }^{67}$ Vorbehaltos opriert Kirchheimer für die zweire Alternative. Aufgabe einer linken Politik sei es, die demokratischen Defizite durch die Veränderung des sozialen status quo abzubauen.

Carl Schmitcs Meinung zur Verfassungsreform ist der Kirchheimers im Grundsätzlichen verblüffend ähnlich. 1930 kritisiert er in einem Redebejurag vor der Mitgliederversammlung des "Langnamvereins", der Interessenvertretung der rheinischen Schwerinduscrie, die Forderung nach einer Reichsreform mit der These, daß jede Reform des Staates norwendig die Alternative oxapitalismus oder Sozialismus* entscheiden müsse. ${ }^{68}$ Den Voren für einen radikalen Bruch mir der Verfassung wird entgegergehalten, daß der staatliche Apparat im Beamtentum und in der Reichswehr über ausreichende Kräfre, die im Sinne der allen Revisionsbestrebungen zugrundeliegenden Optionen zu handeln in der Lage und bereit seien, verfüge. Diese Stürzpunkte gelce es auszubauen, während eine Verfassungszäsur in die Katastrophe führen werde. Zwei Jahre später kann Schmict, nun ais Hzuptreferent,

6) A. 2. O., 5. 96 (S. In Jem 1967 geschricbenen Vorwort zu der Aufsalzsammiung bezcichnet Fraenkel den Vorschlag des -konstrukriven ikiRtmuensvotums- als einen Gedankengang, der sich im Grundge. setz wiederfinde und lür den er dic .erste eingehende schriftlich festgehalecre wissensithateliche Begründung - geliefen habe.

64 Vgl. Heller, Ziele und Grenzen ciner deutschen Vefiassungsreform, in: Neue Bläzter für den Sozialismus, \}. Jg., (1932). S. 376 II.; Simons, Verfassungsreform! ebenda, S. 580 ff.

6s Ders., Verfassungsreform und Sozialdemokratie. FN 33, S. 79 ff. 83.

66 Ders., Die Verfassungsreform. FN 1, S. $9616 ., 96$.

67 FN 69. S. 84 f. Kirchlieimer entwiekele den Gedanken am marxistischen Basis-Uberbau Modell: 8 könne in demokrarischen Gesellschilten der Fall cinereten, daß der ideolugische Uberbau der Rechesnrdnung den earsaehlichen. Macheverhailenissen -voriushinks. Autgahe der Linken sei es dasn. die Machrverhalerisse auf den Sund der Legalordnung zu bringen.

69 Schmitu. Diskussionsbeitrag, in: Mittcilungen der Langnamvercins. Jg. 1910, Nr. to N. F. Heft 19, 5. $45816 ., 4626$. 
vor dem gleichen Gremium die polieischen Aktiva der Zwischenzeir aufzählen.69 Verfassungsreformen seien überflüssig, weil die politischen Kräfre für die Umgestaltung des Staates längst vorhanden seien. Eine neue Verfassung schaife nur neue Legalitäcen und somir neue Schurzrvälle für pluralistische Interessen.

Die Ubereinstimmung der beiden Auroren zeigr sich als nicht bloß in der Ablehnung jeder Verfassungsreform, sondern auch in der Opcion für eine politische Lösung der Krisenerscheinungen. Diamerral entgegengeserze freilich sind die Inhalie der Strategien und dementsprechend die sozialen Kräfte, denen die Umsetzung der strategischen Entwürfe überantwortet wird.

Schmitrs Konzept vertraut auf Beamentum und Reichswehr, die aus einer vorgeblich neutralen Sachlichkeic sowie aus der waus vorpluralistischen Zeiren stammende(n) Auroricär $u^{70}$ des Reichspräsidenten ihre Legitimation beziehen. Erweitert wird die schmale soziale Basis von den Industriellen, denen Schınitr in seinem 2. Langnamvortrag, der unter dem programmarischen Tircl "Gesunde Wirtschafr im starken Stata" in der Zeitschrife *Volk und Reich veröffencliche wurde, eine öffentlich-rechtliche Funkcion überträgt. Die Intervencionen in die Okonomie werden jedem sozialdemokrarisch-gewerkschaftlichen Einfluß entzogen, indem die wirtschaftliche Lenkungsbetugnis eigenständigen Selbstverwaltungskörperschafeen der Industrie anvertraut wird. Die Machtprämienlehre entwickelt die Techniken, die dem envünschren Umbau der Sozial- und Wirtschafrsordnung das Scigma des offenen Verfassungsbruchs ersparen. Zu den supralegalen Prämien auf den legalen Machibesitz's gehör die Komperenz der Auslegung und Anwendung unbestimmer Rechrs- und Ermessensbegrilfe. Gemäß der Logik der Machtprämienlehre vermag die wissenschafcliche Praxis eines anerkannten Staarsrechtlers wie Car\} Schnitr unmitcelbar politische, ja ragespolitische Wirksamkeit zu erlangen, indem der Kanon der jurisrisch vertretbaren Meinungen um die eine machtpolitisch erwünschte Auffassung vermehrt wird. Unter Benfung auf diese in der Literarur vertretene, also vertrecbare Meinung lassen sich dann zunächsi einmal vollendece Tatsachen schaffen. Einige von Schmitts Arbeiten aus den Jahren 19jo bis 193) vermitteln den Eindruck, daß sie in Absprache und zum Zwecke einer vorgezogenen oder nachrräglichen verfassungsrechtlichen Legirimarion des politischen Handeins der Präsidialkabinette geschrieben wurden."

Die sozialen Gruppen, von denen Kirchbeimer die Verteidigung der Republik erwartet, sind die Parteien und Gewerkschakzen der Arbeiterbewegung. Seine politischen Absichten implizieren eine grundsätzliche Kricik der sozialdemokratischen Passivizät und eine nicht weniger scharie Kririk der KPD-Politik. Prinzipie\}l erachter er zwar die Gewalt als ein legitimes Mitcel zur Beseitigung der konstatierten demokratiexidrigen Anomalien der Sozialordnung, jedoch bezieht er die Kriverien für die Gewaltanwendung nicht aus jener marxiscischen Geschichssphilosophie, die das Recht auf die wschöpferische Gewalt" bei dem Proletariat als historisch autsteigender Klasse immer schon gut aufgehoben weiß. Denn die Erfahrungen der russischen Revolution haben Rosa Luxemburgs Leninismuskritik bestärigt und generell das marxistische Konzepi eines unproblematischen Übergangs von der Diktatur des Proletariats in eine proletarische Demokratic massiv in Zweifel

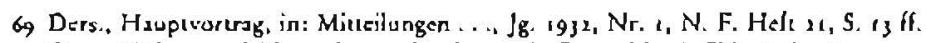

70 Ders, Weiterentwisklung des cotalen Suzes in Deusschland, FN 16, 5. $36 \%$.

7t In: Volk und Reich, Js. 1913.

72 Ders., FN 13, S. 28 , If. Schmir bestimme die Macheprimie als . . . cinen zur bloß nomarivistischlegalen .Mache hinxurrecenden zusäızlichen poliuischen Mehrweet, eine überlegale Prämie aut den lezalen Besirz der legalen Machra. S. 188.

73 Dub̂ diese These keine Untersiellung ist, belegen die nachtraglichen Kommentienungen der Aufsätze jener Periode durch Schmit selbst. Vgl. etwa die Nachbemerkung zu dem Aufsatz FN 70. 
gezogen. Selbst ein Sieg des Prolecariars in einem Offensiv-Bürgerkrieg ist zu teuer erkauft, wenn die proletarische Herrschaftsform nicht imstande ist, .... auch die persönliche Freiheit des Individuums als einen unvergänglichen Bestandteil unserer europäischen Kulcurordnung aufrechtzuerhaiten, "' Die Ausübung proletarischer Gewalt steht demnacls uncer zwei Bedingungen. Zum einen muß die Austragung politischer Konflikte in demokratischen Formen unmöglich geworden sein infolge der Absage des Bürgertums an dic Demokratie. Diese Bedingung siehr Kirchheimer im Jahre r 932 erfüllr, nachdern das deutsche Bürgertum den von Georg Lukács einst der $\Lambda$ rbeiterklasse crteilcen Rat beherzigt und ein mbloß taktisches* Verhälınis zur Legalität eingenommen hat." Die zweite Bedingung nimmt Bezug auf die politische Entwicklung und Reife der Arbeiterklasse. Die Zielsetzung des revolurionären Kamples muß bestimm sein durch das Programm eines demokracischen Sozialismus, der die Notwendigkeit einer festgefügren proletarischen Kamplorganisation zu verbinden weiß mit der Bervahrung der breitesten Vertrauensbasis des ganzen arbeitenden Volkesa. ${ }^{36}$ Dieses Vertrauen gründet in der Gewißheit von der Respektierung der Errungenschaften der bürgerlichen Demokratie in einer nachrevolutionären Gesellschaft. Der Arberteṛbewegung wird die Erhaltung der demokratischen Subscanz der Rcichsverfassung anvertrauc, nachdem das Bürgertum seine eigene Geschichte und politische Kultur verraten hat. Im Gegensatz zu Schmitr, der die Legitimirät der Verfassung reduziert auf einen bürgerlichen Kemgehalt und mit den sozialen Grundrechren die verfassungsgeserzlichen Verbürgungen zugunsten der Arbeiterklasse eliminiert, entrvirft Kirchheimer eine Legitimitär, in der die sozialen Hoffnungen der Arbeiterklasse verschmelzen mit der bürgerlichen Freiheitstradition. Diese Legirimicär, die die Emanzipationschancen der Weimarer Verfassung aufbewahrt, soll verteidigt werden mit außerlegalen, revolutionären Mitteln. Inhalt und Genese der Strategie sind zurückzuführen auf die Erfahrungen jenes Julitages des Jahres 1932, an dem die lezten Weichen für den Untergang der Republik gestellt wurden.

\section{Der Preußenschlage}

Carl Schmitrs Name ist wie der keines anderen deutschen Staatsrechtlers mit der Amrsenthebung der preußischen Regierung und der Bestellung Papens zum Reichskommissar für das Land Preußen durch Verordnung des Reichspräsidenten vom 20. Juli $1932^{77}$ verbunden. Umstriten ist in der Geschichtswissenschaft" lediglich das Ausmaß seiner Mitwirkung an der Vorbereitung des "Preußenschlags“, im Zuge dessen die lezzte von den Parteien der Weimarer Koalition gebildete Länderregierung abgesetzt wurde. Immerhin steht fest, daß Papen in der Kabinetrssitzung vom 2s. Juli Schrnitr als einen der Prozeßvertreter des Reiches im Verlassungsstreitver-

74 Kirchheimer, FN 63, S. 81.

75 G. Lukács, Legalität und IHegalieäe, in: Geschichce und Klassenbewußesein, Berlin 1923. 5. 161 fí., 275.

76 Kirchheimes. FN 99. S. 114.

77 Verordnung des Reichspräsidenten. berreflend die Wiederhersellung der öllentichen Sicheeheir und Ordnung im Gebiet des Landes Preußen, RGBI. I (1932), S. 17.

78 Beacher. FN 60, 5. 381 , berichee von einem Trefien einiger Gefolgsteute Papens und Gayls bes Hindenburg aul Gur Neudeck am I 4 . Juli 1932 und füpr den mil keinem Quellennachweis belogten Sat? an: - Ferner scheint man zur Beurtellung der Begrundungen Carl Schmill beigenogen zu haben, um Hindenburgs Bedenken begegnen zu können-. H. Wuth. Carl Schmiu in der Jeutschen Innenpoluk des Sommers 1931. Beihefr i der HZ, München 1971. S. 103, verweist Jemgegenuber die Annahme Brachers in den Bereich der politischen Legendenbildung. 
Gahren um die Preußenakcion nannte und ankündigte, derselbe werde in einigen Tagen einen staarsrechrlichen Aulsatz, der der Regienung *Recht geben*, werde, publizieren." Jenseits von Spekularionen liegr zudem ein Vorgang, der zumindese darïber Auskunft gibr, daß Schmitt schon vor dem 20. Juli, wenn auch nicht im Auftrage der Reichsregierung, so doch motivien durch deutschnarionale Aufrufe und Publikationen gegen das *schwarz-rore Preußen «, für ein wie immer geartetes Eingreifen gegen die Preußenregierung seine Srimıne erhob. Gerade der seiner Schrift * Legalität und Legitimizär * vorangestellıe, politische $Z$ weckfreiheir suggerierende Vermerk „Diese Abhandlung lag am 10. Juli 1932 abgeschlossen vor « verleiht dem Vorabdruck eines Auszuges aus eben dieser Schrift im zweiten Juliheft der Zeicschrift *Deursches Volkstum eine tagespolitische Dimension. Unter dem Titel - Legalität und gleiche Chance der Machrerringung ${ }^{80}$ wird hier die oben bereits erwähnte Machtprämienlehre in einem eindeurig aktuellen politischen Kontext entwickeit. Sobald zwischen den politischen Parteien die Annahme einer wbeiderseirig gleich legalen Gesinnung* entfalle, werde die im Besitze der legalen Macht befindliche Partei die Machtprämien rücksichrslos ausspielen müssen, um der Schließung der Tür der Legalieät durch den politischen Gegner zuvorzukommen. Exemplifiziert wird die These an der Geschäftsordnungsänderung des preußischen Landrages. Mir deurlicher Anspielung auf die Preußenregierung heißr es weiter, auch das verfassungsmäßig ansonsten unbedenkliche Institut des Geschäftsministeriums sei nicht mehr praktizierbar, weil die interparteiliche Illoyalitär gegenüber der Legalität die Unterscheidung zwischen vlaufenden* und *allen* Geschäfren nicht zulasse. Die Konsequenz ist der Zusammenbruch des Legalitätssysterns, ein »legaljtäts- und verfassungsloser Zustanda. Damit steht das politische System vor der Alernacive einer gewaleförmigen Austragung des policischen Konflikes, also dem Bürgerkrieg, oder der Intervention eines whöheren Drittenw.

Der wenige Tage nach der Preußenakrion in der "Deurschen Jurisren-Zeitung* publizierte Autsatz antizipier das Ergebnis der staatsrechdlichen Úberlegungen im Titel: •Die Verfassungsmäßigkeit der Bestellung eines Reichskommissars für das Land Preußenk. Gerechrferrigr wird das Vorgehen des Reichspräsidenten und der Reichsregienung mir Art. 48 Abs. I und 2 WRV. Die Festscellung der Verletzung der Treuepflichr Preußens gegenüber dem Reich bzw. der erheblichen Störung der öffentlichen Sicherheit und Ordnung in Preußen sei eine präsidiale Ermessensentscheidung, die der justiziellen Uberprüfung entzogen sei. Schmitt verfolgte diese Linie auch im Prozeß vor dem Staalsgerichtshof, in dern er als Vertreter der Reichsregierung auttrat." "Staatsrechtlich betrachret erscheinen seine Ausführungen

79 Muth, 2. 2. O., S. 107 mit Nachweisen. Der zugesagte Aufsatz erschien in Hele is der DJZ vom I. August 1932. Sp. 95) ff. - Die Daten von Sehmitus belegrer Mitwirkung geben Anlab zu Spekulacionen. Dic Vorselllung, die Reichsregierung habe erse nach der PreuBen Akcion die Zuscimmung der als Prozeßverureter lungierenden Juriseren eingeholı und sei damie das Risiko eingegangen, im Prozeß ohne sezaesrectuliche Fürsprecher zu verbleiben, mutet seeniy walirscheinlich an. Wenn aber bereirs vor dem 20. Juli um diese Mitwirkung nachgesuche worden war, ist dann niche anzunehmen. dab die Regienung zugicich Rat fur eine einigermaßen traglähige slaxtsrechetiche Begrundung ihrer Akrion sinholte? Wener wäre zu frazen nach dem Redaksionsschluß von Heft is der DJZ und danach, welche Zeit auch ein Schnellsehreiber wie Sctromitr hür Jie Erarbeitung eincs Aufszezes von soleher Tragweeie benörigle.

so In: Deursches Volkstum, 1932, 2. Julihefh, S. sigif.

81 Der Parecivorstand der SPD hatle die Verhandlungen stenographisch protokollieren lassen. Der Dietz Verlag publizierre die Protokolle nebst cinem Anhang mit Urteil und Dokumenten: Pseußen conera Reish vor Jem Sexatsgericheshof in Leipzig vom 10. bis 14. und vom 17. Okeober 1932, Berlin 1933. Dis Buch aurde 1976 nachgedruckt vom Verlag Detlef Auvermann KG, Glashülten im Taunus. Neben

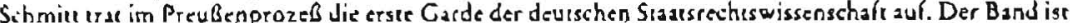
niche nur ein verlassungsgesehín neliches Dokument ersten Ranges, sondern gibl auch einen hervorrabenden Cberblick über die Weimarer Siasurechesschulen. Darüber hinaus wird im Prozeß das 
äußerst problematisch, prozeßtaktisch und politisch aber war die eingenommene Position ungeheuer durchschlagskräftig. Belegt wird die Einschätzung von den beharrlichen Versuchen des Reichsgerichrspräsidenten Bumke, die Stellung und Person Hindenburgs aus dem Prozeß herauszuhalten. ${ }^{82}$ Denn eine Prozeßentscheidung, die die Integrität des Reichspräsidenten tangierte und seine Verfassungsloyalitäc in Zweifel zog, konnte im Herbst 1932 kein deutsches Gericht vertreten. Hindenburg fungierte im - niche nur bürgerlich's - Bewußtsein jener virulenten Zeit als leczte intakte Säule der Weimarer Republik, als Bollwerk gegen die Parteien der Anarchie und des Kulturverfaills. Carl Schmitt jedenfalls durchschaute das Anliegen des Reichsgerichrspräsidenten und stieß in seinem Schlußplädoyer gezielt in die offene Wunde: "Wo ist dieses alles, die Dignität und die Ehre Preußens besser aufgehoben? Bei den am 10. Juli ihres Amres enthobenen geschäftsführenden Ministern, die es dank dem Kunstgriff vorn 12. April noch sind, oder beim Reichspräsidenten von Hindenburg? « $^{84}$

Das in der Hauptsache ergangene Urteil des Statsgerichrshofs vom 25. 10. $193^{211}$ peilte einen Kompromiß an. Die Reichsexekution wurde als verfassungswidrig eingestuft, soweit sie auf Art. $4^{8} \mathrm{Abs}$. r WRV gestützt wurde, da eine Pflichtverlerzung des Landes Preußen gegenüber dem Reich nicht bewiesen werden konnte. Gerechuferrigt wurde das Vorgehen des Reiches mit Ärt, 48 Abs. 2 WRV. Bei der Fessstellung der erheblichen Störung der öffentlichen Sicherheit und Ordnung in Preußen sei weder ein Ermessensmißbrauch noch eine -überschreitung zu verzeichnen, also durfte der Reichspräsident die Aktion für geboten erachten.

Otro Xirchheimer ist auf die mit dem "Preußenschlag aufgerworfenen staatsrechelichen und politischen Fragen in einem Aufsacz in der "Geselischaft a ausführlich eingegangen. ${ }^{86}$ Hier sollen weniger seine verfassungsrechtichen Argumente, die zudem weirgehend nur die Meinungen liberaler Stzarsrechtler zusammenfassen, interessieren als die Einschätzung jener Verfassungslehre, die den Staarsstreich juristisch legitimierte. Dieser Verfassungslehre sei ein ungeheurer Machrzuwachs zugekommen, nachdem die parlamentarische Demokratie in Deurschland ihre soziale Basis verloren habe, andererseirs die gegenseitig sich aufhebenden Kräfre der Parzeien eine Umstrukcurierung der politischen und organisatorischen Grundlagen der Verfassung verunmöglichr hätcen. Deshalb müsse jedoch politische Streitrage auf der Grundlage der Reichsverfassung entschieden werden. Anstatt nun diesen Machtgewinn zur Erhaltung und Stärkung des brüchigen Verfassungskompromisses von 1919 zu nutzen, also - um einen Begriff Schmitrs positiv zu wenden - als "Hüter der Verfassung" zu fungieren, leiste die herrschende Verfassungslehre bei der Demontage der Reichsverfassung durch die Präsidialkabinetre juristische Schürzenhilfe. In Anlehnung an eine gelungene Formulierung in einer venezianischen Gesandrenschrift nennt Kirchheimer das hier grundgelegre W/issenschaftsverständnis nscienza delle circonstance", Wissenschaft der konkreten Umstände." Unausge-

\footnotetext{
Seharnier von Policik, Sratsreche und jusciz in ciner Anschaulichkeit sichrbar, wie es woh! nur in der historischen Ausnahmesituasion des Jahres 1932 möglich war. - Die herausmgende Roile Schmists ise dokumentien in einem Plädoyer Peters, das einer Rezension der Sehmituschen Arbeiten seir 1930 gieichkomnx. S. s6-60.

82 .... ich habe im Verisule des Verhandlungen nachdriellich gebeten, die Person des Herm Reichspräsidenten nach afler Móglichkeit aus dem Spiel zu lassen. Ieh glaube, daß man der Verchning. der Ehrlurcht dem Heren Reıchsprasidenten gegenüber am besten dadurch Ausdruck gibr, daß man ihn in diesem Saale so wenig wic möglich erwähne. A. 2. O., S. 470 .

8) Selbst die Prozeßverterer Preußens hielten die Kanvenienz gegenüber Hindenburg dureh. Vgl. die Aussage Brechis, a. a. O., S. 8.

84 A. a. O., S. ${ }_{469}$.

8, A. 2. O., S. 492 lf

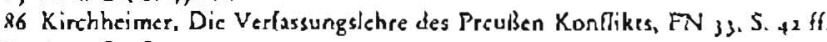

87 A. 2. O.. S. 4 .
} 
sprochen zielt die Kritik suf Schmirt. Kirchheimers Kritik der "Verfassungslehre des Preußen-Konfliktse kommt der Meinung Hellers nahe, der Schmitus Schlußplädoyer vor Jem Stanesgerichtshof mit dem Zwischenruf "Situationsjurisprudenz* unterbrochen hatre. ${ }^{8 B}$

Kirchheimer hacte mit der spötcischen Bemerkung, die apostrophierte Verfassungslehre zeige nur noch Interesse für die wrichrige Arr zu regieren und den Herrschenden zu dienen*, die Indienststellung des Rechts nnter die Geschäfte der Politik treffend charakterisiert. Die Programmatik der Unterwerfung des Rechts war begründet in der stracegischen Unterordnung der Legalitär unter die Legitimirär. Die Legalordnung wurde unter Berufung auf die Legitimitär staatlichen Handelns parciell aufgehoben, die solchermaßen eroberte Machtposition wurde ausgestattec mit der Vermutung der Legalitär, um von dem gesicherten Terrain aus einen neuen Vorstoß anzutreten. Die « Prämien auf den legalen Machtbesizz«, Ermessensausübung, Vermuiung legalen Handelns, soforriger Vollzug, wurden exzessiv ausgeschöpfr. Die Legalität wurde zur Schlinge die sich um den Hals des polizischen Gegners immer fester zuzog. Während die Sozialdemokratie auf die Entscheidung des Sraatsgerichtshofs warete, sorgte Papens Kommissar in Preußen für vollendete Tatsachen. In wenigen Tagen wurden die dernokratisch gesinnten Beamten aus dem Staatsdienst entfernc und die Erfolge, die die Weimarer Koalition in der Demokrarisierung des Staatsaufbaus während der vorangegangenen 12 Jahre erreiche hatce, rückgängig gemachr. Das Agieren der SPD in und nach der Preußenakcion erhellı die von Kirchheimer seit 1928 vorgetragene Kritik der sozialdemokratischen Politik, die oben mit dem unscharfen Begriff „Legalismus» belegt wurde. Legalismus heißr Reduktion von Policik aul die Mitrel der Rechtsordnung in polizischen Situationen, in denen die Macht die Legalität als ein taktisches Herrschafrsnuitcel verwendet. Die SPD verzichtete auf den Generalstreik und vertraute voll und ganz auf die justizförmige Konflikrlösung.

Kirchheimers strategischer Gegenentwurf zur offiziellen Politik der Sozialdemokrarie bedient sich zwar des Schmitrschen Rasters von Legalitäc und Legirimität, er füllt dieses Raster aber nicht bloß aut mit anderen politischen Inhalten. Bei Schmitr ist das Begriffspaar Legalitäc und Legitimitär gespalten bis in die Wurzeln, die geforderte Entscheidung erhält die Dimension einer wletzten Unterscheidung» wie die zwischen Freund und Feind. Kirchbeimer hingegen macht zum einen den Ubergang vom Modell legaler Konflikrlösung zum legitimen revolutionären Handeln abhängig vom Eintritt der politischen Bedingung, daß der Funktionsmechanismus der Legalicät infolge von Änderungen im sozialen Krälreparallelogramm leerläufr. Zum anderen läßr er in die sevolutionäre Legiumität die zu berwahrenden Inhalte des positiven Verfassungsrechts einfließen. Die so konzipierte Legitimität enrwirft ein politisches Programm, das konsenstähig sein muß durch die sozialen Klassen hindurch. Dagegen ist Schmitrs Legitimität inhalrsleer und offen für die beliebigsten politischen Absichten und Taktiken. Kirchheimer hatte 1933 aus der Emigration Schmitts Legitimitätstheorie eingestuft als sdoctrine of the cotalitarian State ${ }^{B y}$ Diese Doktrin sei so inhaltsleer und formal "... . that it equally favoured the Bolshevist and Fascist forms of governmenta. Die verhängnisvolle Olfenheic der Legicimitätsdoktrin wurde bereits in ihrer Geburtsscunde des Preußen Konflikts unter Beweis gestellt. Denn hier ging es von Antang an auch um die Frage der Regierungsiähigkeit der Nacionalsozialiscen. Papen hatte die Abseczung der Preu-

s\& Dukumentier in FN $81,5,{ }_{469}$

s, Nirchieimer. The Growth and the Decay of the Weimar Cunstituston, in: Contemporary Review, Bd.

II. 19נ3. Nr. 815, S. 319 If., 366. 
Ben Regierung $u$, a, begründet mit deren Politik, die dic "sctaatsfeindlichen Kräfte des Kommunismus * mit der waufstrebende(n) Bcwegung der NSDAP * gleichgesetz: hatte. ${ }^{\infty}$ Papens Traum vom "Neuen Staat $\alpha$ als * rocher de bronze * in den Wogen des Panteienhaders war schlechre Ideologie und leichtfercige Politik. Entworfen für die autoritäre Herrschatt einer staarstragenden Elite wurde die Theorie und Praxis des starken Staates zum Einfallstor für nationalsozialistische Machrgelüste.

\section{Die Wege trennen sich}

Reichspräsident v. Hindenburg ernannce am 30. Januar r93s Adoif Hicler zum Reichskanzler. Im Jahre 1972 hat Carl Schmirc, der seit der Vorbereisung des Preußenprozesses als Mann Schleichers im Machrzentrum agierte, eine incime Schilderung der Vorgänge der letzten Januartage vorgelegt.9" Schmitt war ein Gegner der Ernennung Hitlers, er vertrat und unterstüzzte das politische Programm General v. Schleichers, der bereit war, es auf "Hauen und Stechen a ankommen zu lassen, und, nachdem der Plan einer Spalcung der NSDAP und eines Bündnisses $z$ wischen Strassers mlinkem. Parteiflügel und einer Gewerkschaftsachse nicht realisien werden konnce, den Reichstag auflösen, den Staatsnotstand ausrufen und NSDAP und KPD verbieten wollte. Die Militanz des Planes spiegelt sich in Schmitrs Aufsätzen des Winters 1932/33. Das dem Staat reservierte *Monopol des Politischen wird nun gedeuter als *Monopol der $W_{a f f e n}{ }^{91} E_{s}$ ist müßig, über die Erfolgsaussichten des abenteuerlichen Planes zu spekulieren. Die historischen Ereignisse machren solche Pläne zur Makulatur.

Kirchheimers und Schmirts polizische Biographien kreuzten sich noch einmal im Jahre 1935. Kirchheimer lebte damals in Paris und verfaßce für den deurschen Widerstand eine staatsrechcliche Analyse zu dem Thema "Sraatsgefüge und Rechr des dritcen Reichesw." "Die Studie wurde als antifaschissische Kampfschrift in Deutschland verbreitet, wobei die Publikation uneer dem Pseudonym Dr. Hermann Seitz und unter Verwendung der Aulmachung der von Carl Schmitr herausgegebenen Reihe ${ }^{2}$ Der deursche Scaat der Gegenwarta erfolgte." Der Titel der Schrift beziehe sich auf die Publikation Schmirts "Staatsgefüge und Zusammenbruch des

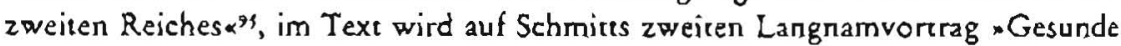
Wirtschaft im starken Staat a angespielt. Kirchhcimer analysiert das Dritze Reich als ein "System gegenseiriger Garantien «, die siegreiche Bürgerkriegspartei Hitlers sei ein partielles Bündnis mit Reichswehr, Sraatsbürokratie und Wirrschaft zum gemeinsamen Zwecke der Herrschafissicherung und Ausbeurung des deurschen Volkes eingegangen. Die Aufteilung des staatlichen Monopols des Politischen ist eine Erkennenis, die auch Schmirts Studie "Staat, Bewegung, Volk ${ }^{96}$ durchziehe. Der Staat als Ämter- und Behördenwesen ist der "politisch - statische Teile der

90 Papen in einer Rundlunkrede vom 20. Juli 1931, zisien nach Fn 81, S. 483 .

91 Vorgerragen hat Schmirt die Schilderung in einem Gespräch mit D. Groh und K. Figge in der Sendung -Zeirgenossena des Südwesilunks Baden-Baden am 6. Februar 1972. 18-19 Uhr. Der Sender hat Jen Texe in einem maschinenschrifelickien Manusk ripe fesegehadeen.

92 Schmit, Machipositionen des modernen States, in: FN 16. S. 367 ff.

9) Abgedruckt in: FN 1, S. Is $2 \mathrm{ff}$, und in: KJ, 1976, S. 44 lf.

94 Uber die Geschiche der Schrifi qurde erstmais berichiet von Herz/Hula, FN i7. S. XVI, Fußnore 16.

Die politischen Hintergründe der Publikation hat W. Luehardt, XJ, 1976. S. 3f ff. ausgeieuchret. ].

Seilere veilte mir dazu mit. daß der Text nach Ansiche von A. R. L. Gurland, rines Freundes von

Kirehbeimer, in Tellen vermurlich von Walli Münzenberg verandert worden ist.

95 In der Reihe: Der deutsehe Staze der Gegenwar, Hefe 6. Hamburg 1934.

96 Ebenda, Hele 1. Hamburg 1933. 
policischen Einheic, die Bewegung deren spolivisch - dynamisches Element $\alpha$, das Volk sei die sim Schutz und Schatren der politischen Entscheidungen wachsende unpolitische Seite" der dreigliedrigen Einheic. Die Formulierungen belegen die Angemessenheit des Anspruchs Kirchheimers, xhinter dem Nebel nationalsozialistischer Phrasen die brutale zynische Wirklichkeit des newen Rechrs zu erkennena. Was man unter dem -deurschen Rechtsstaat Adolf Hirlers « real zu verstehen habe, sei "insbesondere aus den Interpretationen des Professors und Scaatsratsmirglieds Carl Schmitr zu entrehmen " Eine in der "DJZ" abgedruckte Replik" auf Kirchheimers Studie beweist, daB der Schlag getroffen hatre. Der Verfasser Hermann Seitz wird eingeordnet in eine winternationale Verbrecherfront $*$, die versuche, die -geisrige Zusammenarbeir der Völker und damic die Verwirklichung eines echren Friedens zu hintertreibenu. Der Narne des Auroren der Replik wurde nicht genannt. Jedenfalls hat Schmitt als Herausgeber der $\bowtie$ DJZ den Text zu verantworten, gleichguitig, ob er ihn selost verfaße hat. Schmitt scheint den von ihm oft und gern zitieren Sarz Rimbauds: * Le combat spirituel est plus brutal que la bataille d'hommes " verifizieren zu wollen. Brutaler als eine kriegerische Auseinandersezzung wird der Kampl der Argumente, wenn sogar die Einschränkungen und Hegungen, die Schmitrs Freund Feind Theorie dem politischen Feind einräumen, dem wissenschaftlichen Kontrahenten entzogen werden. Denn Verbrecher sind keine ebenbürtigen Gegner, der Kampf gegen sie zielc aut Vernichtung.

Werk und Biographie der beiden Antipoden stehen als Paradigma für die Geschichre der deutschen Inteiligenz dieses Jahrhunderts. Der Sozialist und Jude Kirchheimer mußre emigrieren, erst nach Frankreich und schließlich in die USA, wo er bis zu seinem Tode lebre. ${ }^{\infty}$ Carl Schmits Engagement im Dritten Reich'or und seine rückblickende Selbstinterpretation der Weimarer Arbeiten ${ }^{102}$ nähren die These von der Einheit seiner Theorie. Schmitr geht es insoweit nicht anders als jenen ideengeschichrlichen Strömungen und sozialen Bewegungen der Weimarer Republik, die nichr ausgehend von ihrer Genese, sondern rückblickend von ihrer parriellen Integration in der Gleich- und Selbstgleichschaltungswelle des Jahres r 933 beurteilt werden. Die Schmitt-Sekundärliteratur begründer die Kontinuizärsthese unterschiedlich. Fijalkowski's arbeicet mit einer vorgängigen Option für den Führerstaac, weitaus differenzierter argumentiert Maus ${ }^{\text {sos }}$, die zur Begründung die soziale Funkrion der Theorie heranziehr. Alle Ansärze, die eine über das Jahr 1933 hinausreichende Koncinuität der Theorie Schmitrs annehmen, rekurrieren, soweit sie sich niche mit der naiven Unterstellung verborgener Morive begnügen, auf eine theorieexteme Ebene, auf der das Einheit organisierende Kohäsionsprinzip gefun-

97 Kirchheimer, FN 93. S. I52.

$98 \mathrm{DJZ}$, 40. Jg.. (19,1s). Sp. 1104 f.

9) Schmitt. Clausewitz ais politischer Denker, in: Der Suat, Bd. 6, (1967), S. 479 lf, 502.

100 Kirchheimer arbeirete von $1934-37$ in der Pariser Zweigstelle des Insticuts für Sozialforschung, danach bis $19+2$ in der New Yorker Niederlassung. In den letzen Kriegsjatiren bis in das Jahr 195s arbeikete er an wissenschatelichen Aultragen der amerikanischen Bundesregierung. Von $1955-62$ war er Professor für policische Wrssenschist an der New Sehool for Social Research, Janach bis zu seinem Tode Prolessur an der Columbia Universitär. Naheres im chronolugisehen Lebensiaul in FN I,$S$. XXXIX, und im Nachru! H. Ehmkes, AoR, 91. Bd., S. 117 ff. Gestorben ist Kirchhcimer am 21. 11. 1965, seinem lezzen Wunsch entsprechend wurde seine Asche beigeserzer auf dem Friedhol seiner Geburs. stadt Heilbronn neben dem Grab seiner Eltern.

101 Vgl. dazu meine Arbeit: Der Stase im Bürgerkrigg. Koncinuitäl und Wandlung des Stazesbegrilts in der politischen Theorie Carl Schmius, Frankfurr 1980, insbes. S. $138 \mathrm{ft}$.

102 Sehmile, Positionen und Begrilte im Kampt mit Weimar - Genf - Versailles 1923-1939, Hamburg 1940.

103 J. Fijalkowski, Die Wendung zum Führersta2r, KölnOpladen i958.

10. (. Mrus, Zue -Zisure von 193s in Jer Theorie Call Schmists, in: KJ. 196g, Heft 2, 5. 11) ff.; und: Bürgerliche Rechestheorse und Faschismus. Zut sozialen Funkrion und akiuellen Wiekung der Theorie Carl Schmits, Munchen 1976 
den wird. Die Widersprüche, die cine theorieimmanente Rckonstruktion - überaus deutlich etwa am Beispiel der Schmittschen Rezeption der übelsten Anxisemitismen's - aufwirft, werden als Epiphänomene eines tieferliegenden Geschehens, als unwesentliche diffuse Spiegelungen eines einzigen Fokus begriffen, dessen Ermittlung die ersehnte Einheit verheißs. Meine Bedenken gegen den Begriff der wsozialen Funkciona, der den Fokus in den (sozio) ökonomischen Verllältnissen ertastet, richren sich gegen das ihm eigene redukrionistische Verfahren, das den Blick für Nuancen und Schatrierungen einer Theorie verstellt. Demgegenüber ist $\mathrm{m}$. E. ein Zugang zu den Weimarer Arbeiren Schmitrs fruchcbarer, der die unbestreitbare Nähe der Theorie zur Zeitgeschichre in der Absicht angehr, die begriffliche Verarbeitung politischer Herausforderungen theorieimmanent zu rekonstruieren. ${ }^{10}$ $D_{a} ß$ ein soleher Ansarz Schmitts Engagement im Dritten Reich nicht aus seiner in Weimar entwickeiten Theorie widerspruchsfrei ableiten karn, er vielmehr auf die Kategorie des * Bruches* verwiesen ist, mag itn in den Augen des Ideologiekritikers desavouieren. Gewonnen wird mit der Akzeptanz des Ansatzes eine doppelte Chance. Zum einen läßr er die polisische und intellekruelle Kultur von Weimar selbst sprechen, zum anderen erhält er ein Forschungsfeld für die Frage nach der morbiden Faszinationskraft des Nationalsozialismus auf den relevanten Teil einer Generation deuescher Intellektueller.

Kirchheimers politische Biographie und wissenschaftliches Werk bierer Kriterien an für die Auseinandersetzung mir dem Thema * Inrellekruelle und Machta. Er blieb bis zu seinem frühen Tode der unbestechliche Analyciker der Nahtstellen von Politik, Recht und Justiz, sein Erkenntnisinceresse blieb beseelt von einem konstanten, leidenschafulichen Willen zum Rechrsstaat ${ }^{107}$ und einer humanen Solidarität mir den Opfern der Instrumentalisierung des Rechts durch die Politik. ${ }^{108}$ Seine wissenschaftliche Redlichkeit und politische Integritäc begründen die Hoffnung, daß es möglich ish die sachliche Nähe der $\mathbb{W}$ issenschaft des öffentlichen Rechrs zur Politik auszuhaiten, ohne den Anfechtungen des Politischen in schicksalhafter Unausweichbarkeit ausgeliefert zu sein. Carl Schmitr muß es sich gefallen lassen, an dieser Möglichkeit gemessen zu werden.

10s Die antisemitischen Ausfïlle waren nicht bloB -lip serviec to the teminology of Nationat-Socialist propaganda., wic George Sehwab, The Challenge of the Exception. Berlin 1970. S. 101, allzu wohlmeinend schreibr.

106 Diesen Ansatz habe ich in meiner in FN 101 zitienen Arbejt aus zwei Gründen nichr konsequent durehgehaleen. Einmal hace wh mich dem Schmiessehen $W$ erk über dic $1910-1932$ geschricbencn Arbeiten, die in der Tax uberaus deutliche Bezüge zur Zeitgeschichse aulweisen, angenihers und van daher die Bedeurung des zeirgesehicheliehen Einflusses lür dic vor 1914 und in der ersten Phase von Weimar publizierren Studien ecwas übersehïzzt. Sodann war für mich ein Geschichesverstandnis, das den Abriß hiscorischer Kontinutzäten akzeptien, nicht nachvollziehbar. (Vgl. FN 101, S. 18). Erst dic Arbeiren Foucaules, vor allem das Buch - Archäologie des Wissens, Frankfure 1973-. haben mich getehn, die Kacegorie der -Zäsur- unter anderen als ideologiekrivischen Aspekten zu betrachten.

107 So ureeilt H. Ridder in einem Nachru! aul Kirehheimer, in: NPL. Jg. XIM, (1968), S. 301.

108 Kirchheimers Buch - Polinische Juseiz. trägt die Widmung: Allen Optern der pointischen Justiz in Vergangenheir, Gegenwart und Zukunit. 